\title{
BILATERAL AND BILINEAR GENERATING FUNCTIONS FOR THE MODIFIED GENERALIZED SYLVESTER POLYNOMIALS
}

\author{
Nejla Özmen
}

\begin{abstract}
The present study deals with some new properties for the modified generalized Sylvester polynomials. The results obtained here include various families of multilinear and multilateral generating functions, miscellaneous properties and also some special cases for these polynomials. In addition, we derive a theorem giving certain families of bilateral generating functions for the modified generalized Sylvester polynomials and the generalized Lauricella functions. Finally, we get several interesting results of this theorem.
\end{abstract}

Keywords: Sylvester polynomial, generating function, Lauricella function.

\section{Introduction}

Generalized functions occupy pride of place in literature on special functions. Their importance, which is mounting everyday, stems from the fact that they generalize the well-known one variable special functions, namely, Hermite polynomials, Laguerre polynomials, Legendre polynomials, Gegenbauer polynomials, Jacobi polynomials, Rice polynomials, Generalized Sylvester polynomials, etc. All these polynomials are closely associated with problems of applied nature. For example, Gegenbauer polynomials are deeply connected with axially symmetric potentials in dimensions and contain the Legendre and Chebyshev polynomials as special cases. The hypergeometric functions of which the Jacobi polynomials is a special case are important in many cases of mathematics analysis and its applications.

We define the modified generalized Sylvester polynomials $f_{n}(x ; a, b)$ as follows (see $[10])$ :

$$
f_{n}(x ; a, b)=\frac{(b x)^{n}}{n !}{ }_{2} F_{0}\left[-n, a x ;-;(-b x)^{-1}\right] .
$$

where $b \neq 0$ is an arbitrary constant.

Received November 16, 2017; accepted January 17, 2018

2010 Mathematics Subject Classification. Primary 33C45, 33C47, 33C65 
When $a=1$ and $b=1$ then (1.1) becomes

$$
f_{n}(x ; 1,1)=\phi_{n}(x)
$$

We call the polynomials $f_{n}(x ; a, b)$ modified generalized Sylvester polynomials in view of the relations (1.2). For $a=1$ and $b$ by (1.1) becomes A.K. Agarwal and H.L. Manocha [8] generalization of Sylvester polynomials.

The following generating relations hold for (1.1) (see, [10]):

$$
\begin{aligned}
\sum_{n=0}^{\infty} f_{n}(x ; a, b) t^{n} & =(1-t)^{-a x} e^{b x t} \\
(|t| & <1)
\end{aligned}
$$

and

$$
\sum_{n=0}^{\infty}(\lambda)_{n} f_{n}(x ; a, b) t^{n}=(1-b x t)^{-\lambda}{ }_{2} F_{0}\left[\lambda, a x ;-;\left(\frac{t}{1-b x t}\right)\right],
$$

where ${ }_{2} F_{0}$ denotes Gauss's hypergeometric series whose natural generalization of an arbitrary number of $p$ numerator and $q$ denominator parameters $\left(p, q \in \mathbb{N}_{0}:=\right.$ $\mathbb{N} \cup\{0\})$ is called and denoted by the generalized hypergeometric series ${ }_{p} F_{q}$ defined by

$$
\begin{aligned}
{ }_{p} F_{q}\left[\begin{array}{c}
\alpha_{1}, \ldots, \alpha_{p} ; \quad z \\
\beta_{1}, \ldots, \beta q ;
\end{array}\right] & =\sum_{n=0}^{\infty} \frac{\left(\alpha_{1}\right)_{n} \ldots\left(\alpha_{p}\right)_{n}}{\left(\beta_{1}\right)_{n} \ldots\left(\beta_{q}\right)_{n}} \frac{z^{n}}{n !} \\
& ={ }_{p} F_{q}\left(\alpha_{1}, \ldots, \alpha_{p} ; \beta_{1}, \ldots, \beta q ; z\right)
\end{aligned}
$$

and $(\lambda)_{\nu}$ denotes the Pochhammer symbol defined by

$$
(\lambda)_{0}=1 \text { and }(\lambda)_{\nu}=\frac{\Gamma(\lambda+\nu)}{\Gamma(\lambda)}(\lambda \in \mathbb{C})
$$

in terms of the familiar Gamma function.

Lemma 1.1. The following generating function holds true [2]:

$$
\sum_{n=0}^{\infty}\left(\begin{array}{c}
n+k \\
n
\end{array}\right) f_{n+k}(x ; a, b) t^{n}=(1-t)^{-a x-k} e^{b x t} f_{k}(x ; a, b(1-t))
$$

Proof. If we write $t+u$ instead of $t$ in (1.3), we get

$$
\begin{aligned}
\sum_{n=0}^{\infty} f_{n}(x ; a, b)(t+u)^{n} & =(1-t-u)^{-a x} e^{b x(t+u)} \\
\sum_{n=0}^{\infty} f_{n}(x ; a, b) \sum_{k=0}^{n}\left(\begin{array}{c}
n \\
k
\end{array}\right) t^{n-k} u^{k} & =(1-t)^{-a x}\left(1-\frac{u}{1-t}\right)^{-a x} e^{b x t} e^{b x u} .
\end{aligned}
$$


Replacing $n$ by $n+k$ in the last relation, we may write that

$\sum_{n=0}^{\infty} \sum_{k=0}^{\infty}\left(\begin{array}{c}n+k \\ n\end{array}\right) f_{n+k}(x ; a, b) t^{n} u^{m}=(1-t)^{-a x} e^{b x t} \sum_{k=0}^{\infty}(1-t)^{-k} f_{k}(x ; a, b(1-t)) u^{k}$

From the coefficients of $u^{k}$ on both sides of the equality, one can get the desired result.

Lemma 1.2. The following addition formula holds for the modified generalized Sylvester polynomials $f_{n}(x ; a, b)$ :

$$
f_{n}\left(x_{1}+x_{2} ; a, b\right)=\sum_{m=0}^{n} f_{n-m}\left(x_{1} ; a, b\right) f_{m}\left(x_{2} ; a, b\right) .
$$

Proof. Replacing $x$ by $x_{1}+x_{2}$ in (1.3), we obtain

$$
\begin{aligned}
\sum_{n=0}^{\infty} f_{n}\left(x_{1}+x_{2} ; a, b\right) t^{n} & =(1-t)^{-a x_{1}-a x_{2}} e^{b\left(x_{1}+x_{2}\right) t} \\
& =(1-t)^{-a x_{1}} e^{b x_{1} t}(1-t)^{-a x_{2}} e^{b x_{2} t} \\
& =\sum_{n=0}^{\infty} f_{n}\left(x_{1} ; a, b\right) t^{n} \sum_{m=0}^{\infty} f_{m}\left(x_{2} ; a, b\right) t^{m} \\
& =\sum_{n=0}^{\infty} \sum_{m=0}^{\infty} f_{n}\left(x_{1} ; a, b\right) f_{m}\left(x_{2} ; a, b\right) t^{n+m} \\
& =\sum_{n=0}^{\infty} \sum_{m=0}^{n} f_{n-m}\left(x_{1} ; a, b\right) f_{m}\left(x_{2} ; a, b\right) t^{n} .
\end{aligned}
$$

From the coefficients of $t^{n}$ on both sides of the last equality, one can get the desired result.

The main objective of this paper is to study different properties of the modified generalized Sylvester polynomials. Various families of multilinear and multilateral generating functions, miscellaneous properties and also some special cases for these polynomials are given. In addition, we derive a theorem giving certain families of bilateral generating functions for the modified generalized Sylvester polynomials and the generalized Lauricella functions.

\section{Bilinear and Bilateral Generating Functions}

This section presents several families of bilinear and bilateral generating functions for the modified generalized Sylvester polynomials $f_{n}(x ; a, b)$ given by (1.1) without using Lie algebraic techniques but with the help of a similar method as considered in $[4],[5],[6]$.

We begin by stating the following theorem. 
Theorem 2.1. Corresponding to an identically non-vanishing function $\Omega_{\mu}\left(y_{1}, \ldots, y_{r}\right)$ of $r$ complex variables $y_{1}, \ldots, y_{r}(r \in \mathbb{N})$ and of complex order $\mu, \psi$, let

$$
\Lambda_{\mu, \psi}\left(y_{1}, \ldots, y_{r} ; \zeta\right):=\sum_{k=0}^{\infty} a_{k} \Omega_{\mu+\psi k}\left(y_{1}, \ldots, y_{r}\right) \zeta^{k} \quad\left(a_{k} \neq 0\right)
$$

and

$$
\Theta_{n, p}^{\mu, \psi}\left(x ; a, b ; y_{1}, \ldots, y_{r} ; \xi\right):=\sum_{k=0}^{[n / p]} a_{k} f_{n-p k}(x ; a, b) \Omega_{\mu+\psi k}\left(y_{1}, \ldots, y_{r}\right) \xi^{k} .
$$

Then, for $p \in \mathbb{N}$, we have

$$
\sum_{n=0}^{\infty} \Theta_{n, p}^{\mu, \psi}\left(x ; a, b ; y_{1}, \ldots, y_{r} ; \frac{\eta}{t^{p}}\right) t^{n}=(1-t)^{-a x} e^{b x t} \Lambda_{\mu, \psi}\left(y_{1}, \ldots, y_{r} ; \eta\right)
$$

provided that each member of (2.1) exists.

Proof. For convenience, let $S$ denote the first member of the assertion (2.1) of Theorem 2.1. Then,

$$
S=\sum_{n=0}^{\infty} \sum_{k=0}^{[n / p]} a_{k} f_{n-p k}(x ; a, b) \Omega_{\mu+\psi k}\left(y_{1}, \ldots, y_{r}\right) \eta^{k} t^{n-p k}
$$

Replacing $n$ by $n+p k$, we may write that

$$
\begin{aligned}
S & =\sum_{n=0}^{\infty} \sum_{k=0}^{\infty} a_{k} f_{n}(x ; a, b) \Omega_{\mu+\psi k}\left(y_{1}, \ldots, y_{r}\right) \eta^{k} t^{n} \\
& =\sum_{n=0}^{\infty} f_{n}(x ; a, b) t^{n} \sum_{k=0}^{\infty} a_{k} \Omega_{\mu+\psi k}\left(y_{1}, \ldots, y_{r}\right) \eta^{k} \\
& =(1-t)^{-a x} e^{b x t} \Lambda_{\mu, \psi}\left(y_{1}, \ldots, y_{r} ; \eta\right)
\end{aligned}
$$

which completes the proof.

By using a similar idea, we also get the next result immediately.

Theorem 2.2. Corresponding to an identically non-vanishing function $\Omega_{\mu}\left(y_{1}, \ldots, y_{r}\right)$ of $r$ complex variables $y_{1}, \ldots, y_{r}(r \in \mathbb{N})$ and of complex order $\mu, \psi$, let

$\Lambda_{\mu, \psi}^{n, p}\left(x_{1}+x_{2} ; a, b ; y_{1}, \ldots, y_{r} ; t\right):=\sum_{k=0}^{[n / p]} a_{k} f_{n-p k}\left(x_{1}+x_{2} ; a, b\right) \Omega_{\mu+\psi k}\left(y_{1}, \ldots, y_{r}\right) t^{k}$

where $a_{k} \neq 0, n, p \in \mathbb{N}$ and the notation $[n / p]$ means the greatest integer less than or equal to $n / p$. 
Then, for $p \in \mathbb{N}$, we have

$$
\begin{array}{r}
\sum_{k=0}^{n} \sum_{l=0}^{[k / p]} a_{l} f_{n-k}\left(x_{1} ; a, b\right) f_{k-p l}\left(x_{2} ; a, b\right) \Omega_{\mu+\psi l}\left(y_{1}, \ldots, y_{r}\right) t^{l} \\
=\Lambda_{\mu, \psi}^{n, p}\left(x_{1}+x_{2} ; a, b ; y_{1}, \ldots, y_{r} ; t\right)
\end{array}
$$

provided that each member of (2.2) exists.

Proof. For convenience, let $T$ denote the first member of the assertion (2.2) of Theorem 2.2. Then, upon substituting for the polynomials $f_{n}\left(x_{1}+x_{2} ; a, b\right)$ from the (1.6) into the left-hand side of (2.2), we obtain

$$
\begin{aligned}
T & =\sum_{l=0}^{[n / p]} \sum_{k=0}^{n-p l} a_{l} f_{n-k-p l}\left(x_{1} ; a, b\right) f_{k}\left(x_{2} ; a, b\right) \Omega_{\mu+\psi l}\left(y_{1}, \ldots, y_{r}\right) t^{l} \\
& =\sum_{l=0}^{[n / p]} a_{l}\left(\sum_{k=0}^{n-p l} f_{n-k-p l}\left(x_{1} ; a, b\right) f_{k}\left(x_{2} ; a, b\right)\right) \Omega_{\mu+\psi l}\left(y_{1}, \ldots, y_{r}\right) t^{l} \\
& =\sum_{l=0}^{[n / p]} a_{l} f_{n-p l}\left(x_{1}+x_{2} ; a, b\right) \Omega_{\mu+\psi l}\left(y_{1}, \ldots, y_{r}\right) t^{l} \\
& =\Lambda_{\mu, \psi}^{n, p}\left(x_{1}+x_{2} ; a, b ; y_{1}, \ldots, y_{r} ; t\right)
\end{aligned}
$$

Theorem 2.3. Corresponding to an identically non-vanishing function $\Omega_{\mu}\left(y_{1}, \ldots, y_{r}\right)$ of $r$ complex variables $y_{1}, \ldots, y_{r}(r \in \mathbb{N})$ and of complex order $\mu$, let

$$
\Lambda_{\mu, p, q}\left(x ; a, b ; y_{1}, \ldots, y_{r} ; t\right):=\sum_{n=0}^{\infty} a_{n} f_{m+q n}(x ; a, b) \Omega_{\mu+p n}\left(y_{1}, \ldots, y_{r}\right) t^{n}
$$

where $a_{n} \neq 0$ and

$$
\theta_{n, p, q}\left(y_{1}, \ldots, y_{r} ; z\right):=\sum_{k=0}^{[n / q]}\left(\begin{array}{c}
m+n \\
n-q k
\end{array}\right) a_{k} \Omega_{\mu+p k}\left(y_{1}, \ldots, y_{r}\right) z^{k} .
$$

Then, for $p, q \in \mathbb{N}$; we have

$$
\begin{aligned}
& \sum_{n=0}^{\infty} f_{m+n}(x ; a, b) \theta_{n, p, q}\left(y_{1}, \ldots, y_{r} ; z\right) t^{n} \\
= & (1-t)^{-a x-m} e^{b x t} \Lambda_{\mu, p, q}\left(x ; a, b(1-t) ; y_{1}, \ldots, y_{r} ; z\left(\frac{t}{1-t}\right)^{q}\right)
\end{aligned}
$$

provided that each member of (2.3) exists. 
Proof. For convenience, let $T$ denote the first member of the assertion (2.3) of Theorem 2.3. Then,

$$
T=\sum_{n=0}^{\infty} f_{m+n}(x ; c) \sum_{k=0}^{[n / q]}\left(\begin{array}{c}
m+n \\
n-q k
\end{array}\right) a_{k} \Omega_{\mu+p k}\left(y_{1}, \ldots, y_{r}\right) z^{k} t^{n} .
$$

Replacing $n$ by $n+q k$ and then using (1.5), we may write that

$$
\begin{aligned}
T & =\sum_{n=0}^{\infty} \sum_{k=0}^{\infty}\left(\begin{array}{c}
m+n+q k \\
n
\end{array}\right) f_{m+n+q k}(x ; c) a_{k} \Omega_{\mu+p k}\left(y_{1}, \ldots, y_{r}\right) z^{k} t^{n+q k} \\
& =\sum_{k=0}^{\infty}\left(\sum_{n=0}^{\infty}\left(\begin{array}{c}
m+n+q k \\
n
\end{array}\right) f_{m+n+q k}(x ; a, b) t^{n}\right) a_{k} \Omega_{\mu+p k}\left(y_{1}, \ldots, y_{r}\right)\left(z t^{q}\right)^{k} \\
& =\sum_{k=0}^{\infty} a_{k}(1-t)^{-a x-m-q k} e^{b x t} f_{m+q k}(x ; a, b(1-t)) \Omega_{\mu+p k}\left(y_{1}, \ldots, y_{r}\right)\left(z t^{q}\right)^{k} \\
& =(1-t)^{-a x-m} e^{b x t} \sum_{k=0}^{\infty} a_{k}(1-t)^{-q k} f_{m+q k}(x ; a, b(1-t)) \Omega_{\mu+p k}\left(y_{1}, \ldots, y_{r}\right)\left(z t^{q}\right)^{k} \\
& =(1-t)^{-a x-m} e^{b x t} \Lambda_{\mu, p, q}\left(x ; a, b(1-t) ; y_{1}, \ldots, y_{r} ; z\left(\frac{t}{1-t}\right)^{q}\right)
\end{aligned}
$$

which completes the proof.

\section{Special Cases}

When the multivariable function $\Omega_{\mu+\psi k}\left(y_{1}, \ldots, y_{r}\right), k \in \mathbb{N}_{0}, \quad r \in \mathbb{N}$, is expressed in terms of simpler functions of one and more variables, then we can give further applications of the above theorems. We first set

$$
\Omega_{\mu+\psi k}\left(y_{1}, \ldots, y_{r}\right)=\Phi_{\mu+\psi k}^{(\alpha)}\left(y_{1}, \ldots, y_{r}\right)
$$

in Theorem 2.1, where the multivariable polynomials $\Phi_{\mu+\psi k}^{(\alpha)}\left(x_{1}, \ldots, x_{r}\right)$ [4], generated by

$$
\begin{gathered}
\left(1-x_{1} t\right)^{-\alpha} e^{\left(x_{2}+\ldots+x_{r}\right) t}=\sum_{n=0}^{\infty} \Phi_{n}^{(\alpha)}\left(x_{1}, \ldots, x_{r}\right) t^{n} . \\
\left(\alpha \in \mathbb{C} ;|t|<\left\{\left|x_{1}\right|^{-1}\right\}\right) .
\end{gathered}
$$

We are thus led to the following result which provides a class of bilateral generating functions for the multivariable polynomials $\Phi_{\mu+\psi k}^{(\alpha)}\left(y_{1}, \ldots, y_{r}\right)$ and the modified generalized Sylvester polynomials.

Corollary 3.1. If

$$
\Lambda_{\mu, \psi}\left(y_{1}, \ldots, y_{r} ; \zeta\right):=\sum_{k=0}^{\infty} a_{k} \Phi_{\mu+\psi k}^{(\alpha)}\left(y_{1}, \ldots, y_{r}\right) \zeta^{k} \quad\left(a_{k} \neq 0, \mu, \psi \in \mathbb{C}\right),
$$


then, we have

$$
\begin{aligned}
& \sum_{n=0}^{\infty} \sum_{k=0}^{[n / p]} a_{k} f_{n-p k}(x ; a, b) \Phi_{\mu+\psi k}^{(\alpha)}\left(y_{1}, \ldots, y_{r}\right) \frac{\zeta^{k}}{t^{p k}} t^{n} \\
= & (1-t)^{-a x} e^{b x t} \Lambda_{\mu, \psi}\left(y_{1}, \ldots, y_{r} ; \zeta\right)
\end{aligned}
$$

provided that each member of (3.2) exists.

Remark 3.1. Using the generating relation (3.1) for the multivariable polynomials $\Phi_{n}^{(\alpha)}\left(x_{1}, \ldots, x_{r}\right)$ and getting $a_{k}=1, \mu=0, \psi=1$ in Corollary 3.1, we find that

$$
\begin{aligned}
& \sum_{n=0}^{\infty} \sum_{k=0}^{[n / p]} f_{n-p k}(x ; a, b) \Phi_{k}^{(\alpha)}\left(y_{1}, \ldots, y_{r}\right) \zeta^{k} t^{n-p k} \\
= & (1-t)^{-a x} e^{b x t}\left(1-y_{1} \zeta\right)^{-\alpha} e^{\left(y_{2}+\ldots+y_{r}\right) \zeta} \\
& \left(|\zeta|<\left\{\left|y_{1}\right|^{-1}\right\}, \quad|t|<1\right)
\end{aligned}
$$

If we set $r=1, y_{1}=x_{3}$ and

$$
\Omega_{\mu+\psi k}\left(x_{3}\right)=f_{\mu+\psi k}\left(x_{3} ; a, b\right)
$$

in Theorem 2.2, we have the following bilinear generating functions for the modified generalized Sylvester polynomials.

Corollary 3.2. If

$$
\begin{aligned}
\Lambda_{\mu, \psi}^{n, p}\left(x_{1}+x_{2} ; a, b ; x_{3} ; a, b ; t\right) & :=\sum_{k=0}^{[n / p]} a_{k} f_{n-p k}\left(x_{1}+x_{2} ; a, b\right) f_{\mu+\psi k}\left(x_{3} ; a, b\right) t^{k} \\
\left(a_{k} \quad\right. & \neq 0, \mu, \psi \in \mathbb{C})
\end{aligned}
$$

then, we have

$$
\begin{array}{r}
\sum_{k=0}^{n} \sum_{l=0}^{[k / p]} a_{l} f_{n-k}\left(x_{1} ; a, b\right) f_{k-p l}\left(x_{2} ; a, b\right) f_{\mu+\psi l}\left(x_{3} ; a, b\right) t^{l} \\
=\Lambda_{\mu, \psi}^{n, p}\left(x_{1}+x_{2} ; a, b ; x_{3} ; a, b ; t\right)
\end{array}
$$

provided that each member of (3.3) exists.

Remark 3.2. Taking $a_{l}=1, \mu=0, \psi=1, p=1, t=1$ in Corollary 3.2, we have

$$
\sum_{k=0}^{n} \sum_{l=0}^{k} f_{n-l}\left(x_{1}+x_{2} ; a, b\right) f_{l}\left(x_{3} ; a, b\right)=f_{n}\left(x_{1}+x_{2}+x_{3} ; a, b\right) \text {. }
$$


If we set $s=r$ and

$$
\Omega_{\mu+\psi k}\left(y_{1}, \ldots, y_{r}\right)=u_{\mu+\psi k}^{\left(\alpha_{1}, \ldots, \alpha_{r}\right)}\left(y_{1}, \ldots, y_{r}\right)
$$

in Theorem 2.3, where the Erkus-Srivastava polynomials $u_{n}^{\left(\alpha_{1}, \ldots, \alpha_{r}\right)}\left(y_{1}, \ldots, y_{r}\right)$ is generated by [7],

$$
\begin{gathered}
\prod_{j=1}^{r}\left\{\left(1-x_{j} t^{m_{j}}\right)^{-\alpha_{j}}\right\}=\sum_{n=0}^{\infty} u_{n}^{\left(\alpha_{1}, \ldots, \alpha_{r}\right)}\left(x_{1}, \ldots, x_{r}\right) t^{n} \\
\left(\alpha_{j} \in \mathbb{C}(j=1, \ldots, r) ;|t|<\min \left\{\left|x_{1}\right|^{-1 / m_{1}}, \ldots,\left|x_{r}\right|^{-1 / m_{r}}\right\}\right.
\end{gathered}
$$

we get a family of the bilateral generating functions for the Erkus-Srivastava polynomials and the modified generalized Sylvester polynomials as follows:

\section{Corollary 3.3. If}

$$
\begin{aligned}
\Lambda_{\mu, p, q}\left(x ; a, b ; y_{1}, \ldots, y_{r} ; t\right): & =\sum_{\substack{n=0 \\
\left(a_{n} \neq 0, m \in \mathbb{N}_{0}, \mu, \psi \in \mathbb{C}\right)}}^{\infty} a_{n} f_{m+q n}(x ; a, b) u_{\mu+p n}^{\left(\alpha_{1}, \ldots, \alpha_{r}\right)}\left(y_{1}, \ldots, y_{r}\right) t^{n}
\end{aligned}
$$

and

$$
\theta_{n, p, q}\left(y_{1}, \ldots, y_{r} ; z\right):=\sum_{k=0}^{[n / q]}\left(\begin{array}{c}
m+n \\
n-q k
\end{array}\right) a_{k} u_{\mu+p k}^{\left(\alpha_{1}, \ldots, \alpha_{r}\right)}\left(y_{1}, \ldots, y_{r}\right) z^{k}
$$

where $n, p \in \mathbb{N}$, then we have

$$
\begin{aligned}
& \sum_{n=0}^{\infty} f_{m+n}(x ; a, b) \theta_{n, p, q}\left(y_{1}, \ldots, y_{r} ; z\right) t^{n} \\
= & (1-t)^{-a x-m} e^{b x t} \Lambda_{\mu, p, q}\left(x ; a, b(1-t) ; y_{1}, \ldots, y_{r} ; z\left(\frac{t}{1-t}\right)^{q}\right)
\end{aligned}
$$

provided that each member of (3.4) exists.

Furthermore, for every suitable choice of the coefficients $a_{k}\left(k \in \mathbb{N}_{0}\right)$, if the multivariable functions $\Omega_{\mu+\psi k}\left(y_{1}, \ldots, y_{r}\right), r \in \mathbb{N}$, are expressed as an appropriate product of several simpler functions, the assertions of Theorem 2.1, 2.2, 2.3 can be applied in order to derive various families of multilinear and multilateral generating functions for the family of the modified generalized Sylvester polynomials given explicitly by (1.1). 


\section{Miscellaneous Properties}

In this section we give some properties for the modified generalized Sylvester polynomials $f_{n}(x ; a, b)$ given by $(1.1)$.

Theorem 4.1. The modified generalized Sylvester polynomials $f_{n}(x ; a, b)$ have the following integral representation:

$$
f_{n}(x ; a, b)=\frac{1}{n ! \Gamma(a x)} \int_{0}^{\infty} e^{-u} u^{a x-1}(b x+u)^{n} d u
$$

where, $\operatorname{Re}(a x)>0$.

Proof. If we use the identity

$$
a^{-v}=\frac{1}{\Gamma(v)} \int_{0}^{\infty} e^{-a t} t^{v-1} d t, \quad(\operatorname{Re}(v)>0)
$$

on the left-hand side of the generating function (1.3), we have

$$
\begin{aligned}
\sum_{n=0}^{\infty} f_{n}(x ; a, b) t^{n} & =\frac{1}{\Gamma(a x)} \int_{0}^{\infty} e^{-(1-t) u} u^{a x-1} e^{b x t} d u \\
& =\frac{1}{\Gamma(a x)} \int_{0}^{\infty} e^{-u} u^{a x-1} e^{(b x+u) t} d u \\
& =\frac{1}{\Gamma(a x)} \int_{0}^{\infty} e^{-u} u^{a x-1} \sum_{n=0}^{\infty}(b x+u)^{n} \frac{t^{n}}{n !} d u \\
& =\sum_{n=0}^{\infty}\left(\frac{1}{n ! \Gamma(a x)} \int_{0}^{\infty} e^{-u} u^{a x-1}(b x+u)^{n} d u\right) t^{n}
\end{aligned}
$$

From the coefficients of $t^{n}$ on both sides of the last equality, one can get the desired result.

We now discuss some miscellaneous recurrence relations of the modified generalized Sylvester polynomials. By differentiating each member of the generating function relation (1.3) with respect to $x$ and using

$$
\sum_{n=0}^{\infty} \sum_{k=0}^{\infty} A(k, n)=\sum_{n=0}^{\infty} \sum_{k=0}^{n} A(k, n-k),
$$


we have

$$
\frac{d}{d x} f_{n}(x ; a, b)=b f_{n-1}(x ; a, b)+a \sum_{m=0}^{n-1} \frac{1}{(m+1)} f_{n-m-1}(x ; a, b) .
$$

Besides, by differentiating each member of the generating function relation (1.3) with respect to $t$, we have the following recurrence relation for these polynomials:

$$
(n+1) f_{n+1}(x ; a, b)=x\left(b f_{n}(x ; a, b)+a \sum_{m=0}^{n} f_{n-m}(x ; a, b)\right) .
$$

\section{The Generalized Lauricella Functions}

In the present section, we derive various families of bilateral generating functions for the modified generalized Sylvester polynomials and the generalized Lauricella (or the Srivastava-Daoust) functions. The four Appell functions of two variables, denoted by $F_{1}, F_{2}, \quad F_{3}$ and $F_{4}$ were generalized by Lauricella functions of $n$ variables which are denoted by $F_{A}^{(n)}, F_{B}^{(n)}, F_{C}^{(n)}$ and $F_{D}^{(n)}[2]$ and

$$
F_{A}^{(2)}=F_{2}, \quad F_{B}^{(2)}=F_{3}, F_{C}^{(2)}=F_{4}, \quad F_{D}^{(2)}=F_{1} .
$$

A further generalization of the familiar Kampé de Fériet hypergeometric function in two variables is due to Srivastava and Daoust who defined the generalized Lauricella (or the Srivastava-Daoust ) function as follows [3]:

$$
\begin{aligned}
& F_{C: D^{(1)} ; \ldots ; D^{(n)}}^{A: B^{(1)} ; \ldots, B^{(n)}}\left(\begin{array}{lllll}
{\left[(a): \theta^{(1)}, \ldots, \theta^{(n)}\right]:} & {\left[\left(b^{(1)}\right): \phi^{(1)}\right] ;} & \ldots ; & {\left[\left(b^{(n)}\right): \phi^{(n)}\right] ;} & \\
{\left[(c): \psi^{(1)}, \ldots, \psi^{(n)}\right]:} & {\left[\left(d^{(1)}\right): \delta^{(1)}\right] ;} & \ldots ; & {\left[\left(d^{(n)}\right): \delta^{(n)}\right] ;} & z_{1}, \ldots, z_{n}
\end{array}\right) \\
& =\sum_{m_{1}, \ldots, m_{n}=0}^{\infty} \Omega\left(m_{1}, \ldots, m_{n}\right) \frac{z_{1}^{m_{1}}}{m_{1} !} \ldots \frac{z_{n}^{m_{n}}}{m_{n} !},
\end{aligned}
$$

where, for convenience,

$$
\Omega\left(m_{1}, \ldots, m_{n}\right):=\frac{\prod_{j=1}^{A}\left(a_{j}\right)_{m_{1} \theta_{j}^{(1)}+\ldots+m_{n} \theta_{j}^{(n)}}}{\prod_{j=1}^{C}\left(c_{j}\right)_{m_{1} \psi_{j}^{(1)}+\ldots+m_{n} \psi_{j}^{(n)}}} \frac{\prod_{j=1}^{B^{(1)}}\left(b_{j}^{(1)}\right)_{m_{1} \phi_{j}^{(1)}}}{\left.\prod_{j}^{(1)}\right)_{m_{1} \delta_{j}^{(1)}}} \ldots \frac{\prod_{j=1}^{B^{(n)}}\left(b_{j}^{(n)}\right)_{m_{n} \phi_{j}^{(n)}}}{\prod_{j=1}^{(n)}\left(d_{j}^{(n)}\right)_{m_{n} \delta_{j}^{(n)}}}
$$

the coefficients

$$
\begin{aligned}
& \theta_{j}^{(k)}(j=1, \ldots, A ; k=1, \ldots, n) \text { and } \phi_{j}^{(k)}\left(j=1, \ldots, B^{(k)} ; k=1, \ldots, n\right), \\
& \psi_{j}^{(k)}(j=1, \ldots, C ; k=1, \ldots, n) \text { and } \delta_{j}^{(k)}\left(j=1, \ldots, D^{(k)} ; k=1, \ldots, n\right)
\end{aligned}
$$


are real constants and $\left(b_{B^{(k)}}^{(k)}\right)$ abbreviates the array of $B^{(k)}$ parameters

$$
b_{j}^{(k)}\left(j=1, \ldots, B^{(k)} ; k=1, \ldots, n\right)
$$

with similar interpretations for other sets of parameters [1]. Here, as usual, $(\lambda)_{v}$ denotes the Pochhammer symbol.

For a suitably bounded non-vanishing multiple sequence $\left\{\Omega\left(m_{1}, m_{2}, \ldots, m_{s}\right)\right\}_{m_{1}, \ldots, m_{s} \in \mathbb{N}_{0}}$ of real or complex parameters, let $\varphi_{n}\left(u_{1} ; u_{2}, \ldots, u_{s}\right)$ of $s$ (real or complex) variables $u_{1} ; u_{2}, \ldots, u_{s}$ defined by $[1]$

$$
\begin{aligned}
\varphi_{n}\left(u_{1} ; u_{2}, \ldots, u_{s}\right): & =\sum_{m_{1}=0}^{n} \sum_{m_{2}, \ldots, m_{s}=0}^{\infty} \frac{(-n)_{m_{1}}((b))_{m_{1} \phi}}{((d))_{m_{1} \delta}} \\
& \times \Omega\left(f\left(m_{1}, \ldots, m_{s}\right), m_{2}, \ldots, m_{s}\right) \frac{u_{1}^{m_{1}}}{m_{1} !} \ldots \frac{u_{s}^{m_{s}}}{m_{s} !}
\end{aligned}
$$

where, for convenience,

$$
((b))_{m_{1} \phi}=\prod_{j=1}^{B}\left(b_{j}\right)_{m_{1} \phi_{j}} \text { and }((d))_{m_{1} \delta}=\prod_{j=1}^{D}\left(d_{j}\right)_{m_{1} \delta_{j}} .
$$

Theorem 5.1. The following bilateral generating function holds true:

$$
\begin{aligned}
& \sum_{n=0}^{\infty} f_{n}(x ; a, b) \varphi_{n}\left(u_{1} ; u_{2}, \ldots, u_{s}\right) t^{n} \\
= & (1-t)^{-a x} e^{b x t} \sum_{m_{1}, k, m_{2}, \ldots, m_{s}=0}^{\infty} \frac{((b))_{\left(m_{1}+k\right) \phi}(a x)_{k}}{((d))_{\left(m_{1}+k\right) \delta}} \\
& \times \Omega\left(f\left(\left(m_{1}+k\right), \ldots, m_{s}\right), m_{2}, \ldots, m_{s}\right) \frac{\left(-u_{1} b x t\right)^{m_{1}}}{m_{1} !} \frac{\left(\frac{u_{1} t}{t-1}\right)^{k}}{k !} \frac{u_{2}^{m_{2}}}{m_{2} !} \ldots \frac{u_{s}^{m_{s}}}{m_{s} !},
\end{aligned}
$$

where $\varphi_{n}\left(u_{1} ; u_{2}, \ldots, u_{s}\right)$ is given by (5.1).

Proof. By using the relationship (1.5), it is easily observed that

$$
\begin{aligned}
& \sum_{n=0}^{\infty} f_{n}(x ; a, b) \varphi_{n}\left(u_{1} ; u_{2}, \ldots, u_{s}\right) t^{n} \\
= & \sum_{n=0}^{\infty} f_{n}(x ; a, b) \sum_{m_{1}=0}^{n} \sum_{m_{2}, \ldots, m_{s}=0}^{\infty} \frac{(-n)_{m_{1}}((b))_{m_{1} \phi}}{((d))_{m_{1} \delta}} \\
\times & \Omega\left(f\left(m_{1}, \ldots, m_{s}\right), m_{2}, \ldots, m_{s}\right) \frac{u_{1}^{m_{1}}}{m_{1} !} \ldots \frac{u_{s}^{m_{s}}}{m_{s} !} t^{n} \\
= & \sum_{m_{1}, m_{2}, \ldots, m_{s}=0}^{\infty} \frac{((b))_{m_{1} \phi}}{((d))_{m_{1} \delta}} \Omega\left(f\left(m_{1}, \ldots, m_{s}\right), m_{2}, \ldots, m_{s}\right)
\end{aligned}
$$




$$
\begin{gathered}
\times\left(-u_{1} t\right)^{m_{1}} \frac{u_{2}^{m_{2}}}{m_{2} !} \ldots \frac{u_{s}^{m_{s}}}{m_{s} !}(1-t)^{-a x-m_{1}} e^{b x t} f_{m_{1}}(x ; a, b(1-t)) \\
=(1-t)^{-a x} e^{b x t} \sum_{m_{1}, m_{2}, \ldots, m_{s}=0}^{\infty} \frac{((b))_{m_{1} \phi}}{((d))_{m_{1} \delta}} \Omega\left(f\left(m_{1}, \ldots, m_{s}\right), m_{2}, \ldots, m_{s}\right) \\
\quad \times\left(-\frac{u_{1} t}{1-t}\right)^{m_{1}} \frac{u_{2}^{m_{2}}}{m_{2} !} \ldots \frac{u_{s}^{m_{s}}}{m_{s} !} \frac{(b x(1-t))^{m_{1}}}{m_{1} !} \sum_{k=0}^{m_{1}}\left(-m_{1}\right)_{k}(a x)_{k} \frac{(-b x(1-t))^{-k}}{k !} \\
=(1-t)^{-a x} e^{b x t} \quad \times \quad \frac{\left(-u_{1} b x t\right)^{m_{1}}}{m_{1} !} \frac{\left(\frac{u_{1} t}{t-1}\right)^{k}}{k !} \frac{u_{2}^{m_{2}}}{m_{2} !} \ldots \frac{u_{s}^{m_{s}}}{m_{s} !} .
\end{gathered}
$$

By appropriately choosing the multiple sequence $\Omega\left(m_{1}, m_{2}, \ldots, m_{s}\right)$ in Theorem 5.1 , we obtain several interesting results as follows which give bilateral generating functions for the generalized Sylvester polynomials and the generalized Lauricella (or the Srivastava-Daoust) functions.

I.By letting

$$
\begin{aligned}
& \Omega\left(f\left(m_{1}, \ldots, m_{s}\right), m_{2}, \ldots, m_{s}\right) \\
= & \frac{\prod_{j=1}^{A}\left(a_{1 j}\right)_{m_{1} \theta_{j}^{(1)}+\ldots+m_{s} \theta_{j}^{(s)}}}{\prod_{j=1}^{B^{(2)}\left(b_{j}^{(2)}\right)_{m_{2} \phi_{j}^{(2)}}} \ldots \frac{\prod_{j=1}^{B^{(s)}}\left(b_{j}^{(s)}\right)_{m_{s} \phi_{j}^{(s)}}}{\prod_{j=1}^{D^{(2)}}\left(c_{j}\right)_{m_{1} \psi_{j}^{(1)}}+\ldots+m_{s} \psi_{j}^{(s)}} \prod_{j=1}^{(s)}\left(d_{j}^{(2)}\right)_{m_{2} \delta_{j}^{(2)}}} \prod_{j=1}^{(s)}\left(d_{j}^{(s)}\right)_{m_{s} \delta_{j}^{(s)}}
\end{aligned}
$$

in Theorem 5.1, we obtain the following result.

Corollary 5.1. The following bilateral generating function holds true:

$$
\begin{aligned}
& \sum_{n=0}^{\infty} f_{n}(x ; a, b) F_{E: D ; D^{(2)} ; \ldots ; D^{(s)}}^{A: B+1 ; B^{(2)} ; \ldots ; B^{(s)}} \\
& \left(\begin{array}{llllll}
{\left[\left(a_{1}\right): \theta^{(1)}, \ldots, \theta^{(s)}\right]:} & {[-n: 1],} & {[(b): \phi] ;} & {\left[\left(b^{(2)}\right): \phi^{(2)}\right] ;} & \ldots ; & {\left[\left(b^{(s)}\right): \phi^{(s)}\right] ;} \\
{\left[(c): \psi^{(1)}, \ldots, \psi^{(s)}\right]:} & {[(d): \delta] ;} & {\left[\left(d^{(2)}\right): \delta^{(2)}\right] ;} & \ldots ; & {\left[\left(d^{(s)}\right): \delta^{(s)}\right] ;}
\end{array}\right. \\
& =(1-t)^{-a x} e^{b x t} F_{E+D: 0 ; 0 ; D^{(2)} ; \ldots ; D^{(s)}}^{A+B: 0 ; 1 ; B^{(2)} ; \ldots ; B^{(s)}} \\
& \left(\begin{array}{ccccccc}
{\left[(e): \varphi^{(1)}, \ldots, \varphi^{(s+1)}\right]:} & -, & {[a x: 1] ;} & {\left[\left(b^{(2)}\right): \phi^{(2)}\right] ;} & \ldots ; & {\left[\left(b^{(s)}\right): \phi^{(s)}\right]} \\
{\left[(f): \xi^{(1)}, \ldots, \xi^{(s+1)}\right]:} & - & -; & {\left[\left(d^{(2)}\right): \delta^{(2)}\right] ;} & \ldots ; & {\left[\left(d^{(s)}\right): \delta^{(s)}\right]}
\end{array}\right. \\
& \left.u_{1}, u_{2}, \ldots, u_{s}\right) t^{n}
\end{aligned}
$$




$$
\left.\left(-u_{1} b x t\right),\left(\frac{u_{1} t}{t-1}\right), u_{2}, \ldots, u_{s}\right)
$$

where the coefficients $e_{j}, f_{j}, \varphi_{j}^{(s)}$ and $\xi_{j}^{(s)}$ are given by

$$
\begin{gathered}
e_{j}=\left\{\begin{array}{cc}
a_{1 j}, & (1 \leq j \leq A) \\
b_{j-A}, & (A<j \leq A+B)
\end{array}\right. \\
f_{j}=\left\{\begin{array}{cc}
c_{j}, & (1 \leq j \leq E) \\
d_{j-E}, & (E<j \leq E+D)
\end{array}\right. \\
\varphi_{j}^{(r)}=\left\{\begin{array}{cc}
\theta_{j}^{(1)} & (1 \leq j \leq A ; 1 \leq r \leq 2) \\
\theta_{j}^{(r-1)} & (1 \leq j \leq A ; 2<r \leq s+1) \\
\phi_{j-A} & (A<j \leq A+B ; 1 \leq r \leq 2) \\
0 & (A<j \leq A+B ; 2<r \leq s+1)
\end{array}\right.
\end{gathered}
$$

and

$$
\xi_{j}^{(r)}=\left\{\begin{array}{cc}
\psi_{j}^{(1)} & (1 \leq j \leq E ; 1 \leq r \leq 2) \\
\psi_{j}^{(r-1)} & (1 \leq j \leq E ; 2<r \leq s+1) \\
\delta_{j-E} & (E<j \leq E+D ; 1 \leq r \leq 2) \\
0 & (E<j \leq E+D ; 2<r \leq s+1)
\end{array}\right.
$$

respectively.

II. Upon setting

$$
\Omega\left(f\left(m_{1}, \ldots, m_{s}\right), m_{2}, \ldots, m_{s}\right)=\frac{\left(a_{1}\right)_{m_{1}+\ldots+m_{s}}\left(b_{2}\right)_{m_{2}} \ldots\left(b_{s}\right)_{m_{s}}}{\left(c_{1}\right)_{m_{1}} \ldots\left(c_{s}\right)_{m_{s}}}
$$

and

$$
\phi=\delta=0 \quad\left(\text { that is, } \phi_{1}=\ldots=\phi_{B}=\delta_{1}=\ldots=\delta_{D}=0\right)
$$

in Theorem 5.1, we obtain the following result.

Corollary 5.2. The following bilateral generating function holds true:

$$
\begin{aligned}
& \sum_{n=0}^{\infty} f_{n}(x ; a, b) F_{A}^{(s)}\left[a_{1},-n, b_{2}, \ldots, b_{s} ; c_{1}, \ldots, c_{s} ; u_{1}, u_{2}, \ldots, u_{s}\right] t^{n} \\
& =(1-t)^{-a x} e^{b x t} F_{1: 0 ; 0 ; 1 ; \ldots ; 1}^{1: 0 ; 1 ; 1 ; \ldots ; 1} \\
& \left(\begin{array}{cccccc}
{\left[\left(a_{1}\right): 1, \ldots, 1\right]:} & -; & {[a x: 1] ;} & {\left[b_{2}: 1\right] ;} & \ldots ; & {\left[b_{s}: 1\right] ;} \\
{\left[\left(c_{1}\right): \psi^{(1)}, \ldots, \psi^{(s+1)}\right]:} & -; & -; & {\left[c_{2}: 1\right] ;} & \ldots ; & {\left[c_{s}: 1\right] ;}
\end{array}\right. \\
& \left.\left(-u_{1} b x t\right),\left(\frac{u_{1} t}{t-1}\right), u_{2}, \ldots, u_{s}\right),
\end{aligned}
$$


where the coefficients $\psi^{(\eta)}$ are given by

$$
\psi^{(\eta)}=\left\{\begin{array}{l}
1, \quad(1 \leq \eta \leq 2) \\
0, \quad(2<\eta \leq s+1)
\end{array}\right.
$$

and $F_{A}^{(s)}$ is the first kind of Lauricella functions.

III. If we put

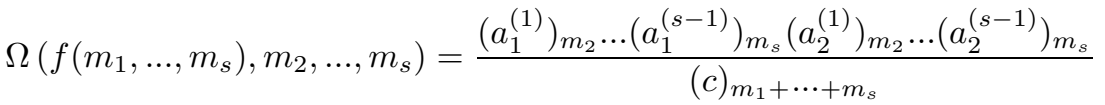

and

$$
B=1, \phi_{1}=1 \text { and } \delta=0
$$

in Theorem 5.1, we obtain the following result.

Corollary 5.3. The following bilateral generating function holds true:

$$
\begin{aligned}
& \sum_{n=0}^{\infty} f_{n}(x ; a, b) F_{B}^{(s)}\left[-n, a_{1}^{(1)}, \ldots, a_{1}^{(s-1)}, b_{1}, a_{2}^{(1)}, \ldots, a_{2}^{(s-1)} ; c ; u_{1}, u_{2}, \ldots, u_{s}\right] t^{n} \\
& =(1-t)^{-a x} e^{b x t} F_{1: 0 ; 0 ; 0 ; \ldots ; 0}^{1: 0 ; 1 ; 2 ; \ldots ; 2} \\
& \left(\begin{array}{cccccc}
{\left[\left(b_{1}\right): \theta^{(1)}, \ldots, \theta^{(s+1)}\right]:} & -; & {[a x: 1] ;} & {\left[a^{(1)}: 1\right] ;} & \ldots ; & {\left[a^{(s-1)}: 1\right]} \\
{[(c): 1, \ldots, 1]:} & -; & -; & -; & \ldots ; & -;
\end{array}\right. \\
& \left.\left(-u_{1} b x t\right),\left(\frac{u_{1} t}{t-1}\right), u_{2}, \ldots, u_{s}\right)
\end{aligned}
$$

where the coefficients $\theta^{(\eta)}$ are given by

$$
\theta^{(\eta)}=\left\{\begin{array}{l}
1, \quad(1 \leq \eta \leq 2) \\
0, \quad(2<\eta \leq k+1)
\end{array}\right.
$$

and $F_{B}^{(s)}$ is the second kind of Lauricella functions.

IV.By letting

$$
\Omega\left(f\left(m_{1}, \ldots, m_{s}\right), m_{2}, \ldots, m_{s}\right)=\frac{\left(a_{1}\right)_{m_{1}+\ldots+m_{s}}\left(b_{2}\right)_{m_{2}} \ldots\left(b_{s}\right)_{m_{s}}}{(c)_{m_{1}+\ldots+m_{s}}}
$$

and

$$
\phi=\delta=0,
$$

in Theorem 5.1, we obtain the following result. 
Corollary 5.4. The following bilateral generating function holds true:

$$
\begin{aligned}
& \sum_{n=0}^{\infty} f_{n}(x ; a, b) F_{D}^{(s)}\left[a_{1},-n, b_{2}, \ldots, b_{s} ; c ; u_{1}, u_{2}, \ldots, u_{s}\right] t^{n} \\
= & (1-t)^{-a x} e^{b x t} F_{D}^{(s+1)}\left[a_{1}, 0, a x, b_{2}, \ldots, b_{s} ; c ;\left(-u_{1} b x t\right),\left(\frac{u_{1} t}{t-1}\right), u_{2}, \ldots, u_{s}\right]
\end{aligned}
$$

and $F_{D}^{(s)}$ is the forth kind of Lauricella functions.

\section{REF EREN C ES}

1. S.-J. Liu, S.-D. Lin, H. M. SRivastava and M.-M. Wong, Bilateral generating functions for the Erkus-Srivastava polynomials and the generalized Lauricella functions, App. Mathematcis and Comp., 218(2012), 7685-7693.

2. H. M. SRivastava and H. L. Manocha, A Treatise on Generating Functions, Halsted Press (Ellis Horwood Limited, Chichester), John Wiley and Sons, New York, 1984 .

3. H. M. SRIVAstava and M. C. DaOust, Certain generalized Neumann expansions associated with the Kampé de Fériet function, Nederl. akad. Westensch. Indag. Math. 31(1969), 449-457.

4. N. Özmen Rm And E. ERkus-Duman, Some results for a family of multivariable polynomials, AIP Conference Proceedings, 1558(1), 1124(2013).

5. N. Özmen and E. Erkus-Duman, On the Poisson-Charlier polynomials, Serdica Math. J., 41(2015), 457-470.

6. N. Özmen and E. Erkus-Duman, Some families of generating functions for the generalized Cesáro polynomials, J. Comput. Anal. Appl., 25(4)(2018), 670-683.

7. E. Erkuş and H. M. SRIVAstava, A unified presentation of some families of multivariable polynomials, Integral Transform Spec. Funct., 17(2006), 267-273.

8. A. K. Agarwal and H. L. Manocha, On some new generating functions, Indian J.Pure App. Math., 13(1984), 1369-1375.

9. A. K. Agarwal, A note on generalized Sylvester Polynomials, Indian J.Pure App. Math. 15(4)(1982), 431-434.

10. M. A. MALIK, A Note on Generating Functions and Summation Formulae for Modified Generalized Sylvester Polynomials, International Journal on Recent and Innovation Trends in Computing and Communication, 2(2014), 2162-2165.

Nejla Özmen

Düzce University

Faculty of Art and Science

Department of Mathematics

Konuralp TR-81620, Düzce, Turkey

E-mail address: nejlaozmen06@gmail.com, nejlaozmen@duzce.edu.tr 